\title{
The sensitivity of BRCA1 mutation carriers to ionising radiation: questions of methodology
}

\author{
Magdalena Chechlinska $\cdot$ Radoslawa Nowak
}

Published online: 27 May 2008

(C) Springer Science+Business Media, LLC. 2008

To the Editor

In a recently published paper in your journal, Gronwald et al. [1] set out to establish whether breast cancer risk in $B R C A 1$ gene mutation carriers relates to early chest X-ray exposures. As shown earlier by Andrieu et al. [2], any reported exposure to chest $\mathrm{X}$-rays is associated with an increased risk of breast cancer in $B R C A$ mutation carriers. Gronwald et al. intended to confirm these findings in the Polish population. The researchers assumed that "women with breast cancer would be more likely to recall or report an early X-ray than women without breast cancer (recall bias)"; thus, in order to avoid this bias, they decided to examine women with breast cancer but with no BRCA1 mutation as a reference group of better relevance. In other words, the reported number of X-ray exposures was compared in breast cancer patients with and without BRCAl mutation. Based on this comparison, it was concluded that "women with a BRCAl mutation may be more sensitive than non-carriers to the effects of ionising radiation, in terms of breast cancer risk". It is a fallacy, and there are no grounds for this conclusion. By comparing BRCAl mutation carriers with non-carriers, both with breast cancer, one cannot draw any conclusions about the influence of any factor on breast cancer risk in the carriers. The appropriate control group in this study is lacking.

The only valid conclusion that might be drawn from this comparative study would be that BRCAl mutation carriers, for unknown reasons, have had more frequent early age $\mathrm{X}$-ray examination, or that they were more likely to report $\mathrm{X}$-ray exposure, trying (perhaps subconsciously) to explain their weak position as a mutation carrier (recall bias). According to Gronwald et al. there is no reason for the recall bias related to the mutation status. Instead, the researchers discuss the question of a family history of breast cancer, which is in fact a minor issue in this study, as $73 \%$ of the affected carriers had no family history of breast cancer.

The appropriate conclusive methodological setting for studying the influence of radiation exposure on breast cancer onset in BRCA mutation carriers is that applied by Andrieu et al., i.e. a comparison between BRCA mutation carriers with and without cancer.

\section{References}

1. Gronwald J, Pijpe A, Byrski T et al (2008) Early radiation exposures and BRCA1-associated breast cancer in young women from Poland. Breast Cancer Res Treat. doi:10.1007/s10549-008-9892-9

2. Andrieu N, Easton DF, Chang-Claude J et al (2006) Effect of chest $\mathrm{X}$-rays on the risk of breast cancer among BRCA1/2 mutation carriers in the international BRCA1/2 carrier cohort study: a report from the EMBRACE, GENEPSO, GEO-HEBON, and IBCCS Collaborators' Group. J Clin Oncol 24:3361-3366
M. Chechlinska $(\bowtie) \cdot R$. Nowak

Department of Immunology, Maria Sklodowska-Curie Memorial

Cancer Centre and Institute of Oncology, Roentgena 5,

02-781 Warsaw, Poland

e-mail: chech@coi.waw.pl 